\title{
Adminstration of PEG-
}

\section{Interferon to a Patient with}

Ulcerative Colitis and Chronic

Hepatitis C Correlated with

Reduced Colonic Inflammation

and Reversal of Peripheral

Th1/Th2 Ratios

\author{
Katsuhiro Kasahara Hiroshi Nakase Norimitsu Uza \\ Satoru Ueno Minoru Matsuura Sakae Mikami \\ Satoko Inoue Tsutomu Chiba \\ Department of Gastroenterology and Hepatology, Graduate School of Medicine, \\ Kyoto University, Kyoto, Japan
}

\section{Key Words}

Interferon · Ulcerative colitis · Intracellular cytokine $\cdot$ Th1/Th2 ratios

\begin{abstract}
A 42-year-old man with chronic hepatitis $C$ and ulcerative colitis (UC) was referred to our hospital in August 2004 because of bloody diarrhea. He was clinically and endoscopically diagnosed with flare of UC. After informed consent had been obtained, he was treated with PEG-IFN- $a-2 a$. Four weeks after initiation of PEG-IFN therapy, his abdominal symptoms gradually subsided. Intracellular cytokine assay revealed that the ratio of Thelper (Th) 1 (IFN- $\gamma$ )/Th 2 (IL-4) increased after IFN therapy. Three months after starting IFN therapy, colonoscopy revealed a normal mucosal pattern. He was uneventfully treated with PEG-IFN- $a-2$ a for one year. When last seen in November 2006, he was still in remission of UC. Our intracellular cytokine data suggested that alteration of Th1/Th2 cytokine balance by IFN is one possible mechanisms of reducing intestinal inflammation in patients with UC. In this regard, IFN therapy could be useful for some patients with UC refractory to other conventional therapies.
\end{abstract}

Several clinical trials have been performed to investigate the therapeutic effect and safety of IFN on patients with UC. Bargiggia and colleagues reported that IFN- $\alpha$ can be safely administered to patients with chronic hepatitis $\mathrm{C}$ and inflammatory bowel disease 
[1]. Madsen and colleagues also reported that IFN- $\alpha 2 a$ treatment resulted in significant depression of the disease activity of ulcerative colitis (UC) [2]. On the other hand, recombinant IFN $\beta$ - 1 a was safe but not significantly effective for steroid-refractory UC [3]. Thus, clinical outcome of treatments with IFN for patients with UC is inconsistent depending on the dose, duration of therapy, and types of IFNs. Therefore, the effects of IFN- $\alpha$ on patients with UC are still controversial. We report here the case of an UC patient with chronic hepatitis $\mathrm{C}$ who was successfully treated with PEG-IFN- $\alpha-2 \mathrm{a}$.

\section{Case Report}

A 42-year-old man with HCV was referred to our hospital in August 2004 because of diarrhea and bloody stool. Ten years before admission, he had been diagnosed with total UC endoscopically and histologically. He had been treated with 3,000 mg mesalazine alone. Physical examination on admission showed mild tenderness in the lower abdomen. Colonoscopic examination revealed reddish and edematous mucosa with multiple erosions through the entire colon (fig. 1a). Disease activity index (Sutherland Index) was scored as 8. Laboratory data showed thrombocytopenia (57,000/mm3), asparate aminotransferase (AST) $124 \mathrm{IU} / \mathrm{l}$, alanine aminotransferase (ALT) $110 \mathrm{IU} / \mathrm{l}$, and hepatitis C virus (HCV) RNA (genotype Ib) $757 \mathrm{kIU} / \mathrm{ml}$. Liver biopsy revealed liver injury associated with HCV (F3/A2). The patient refused both administration of corticosteroid and leukocytapheresis therapy. After informed consent had been obtained, he was treated with PEG-IFN- $\alpha-2 \mathrm{a}(90 \mu \mathrm{g} / \mathrm{week})$ for improving both colonic and liver inflammation. Four weeks after initiation of PEG-IFN therapy, his abdominal symptoms gradually subsided. We performed intracellular cytokine assay with peripheral CD4 T cells before and after IFN therapy. The ratio of T-helper (Th) 1 (IFN- $\gamma$ )/Th 2 (interleukin (IL)-4) increased (40.2) at 4 weeks after IFN therapy compared to that before (27.4) (fig. $2 \mathrm{a}, \mathrm{b}$ ). However, there was no significant difference of IL-10 production from CD4 T cells between before and after IFN therapy. Three months after starting IFN therapy, colonoscopy revealed normal mucosal pattern (fig. 1b). DAI was scored as 2 . He was uneventfully treated with PEG-IFN- $\alpha-2$ a for one year. When last seen in November 2006, he was still in remission of UC and his laboratory data showed a negative serum HCVRNA, which was suggestive of sustained virological response.

\section{Discussion}

INF- $\alpha$, through its immunomodulatory function, could have an impact on pathways in the immune system [4]. Therefore, the effect of INF- $\alpha$ on the pathophysiology of IBD has been focused, because patients with IBD are characterized by imbalance of the Th1/Th2 cytokine response.

Previous reports showed that a treatment with INF- $\alpha$ might be a trigger for development of Th1-related intestinal disease such as celiac disease and Crohn's disease, because INF- $\alpha$ plays an important role in T cell differentiation towards a Th1 type of immune response $[5,6]$. Generally, the pathophysiology of $U C$ is associated with a Th2 phenotype. In this regard, INF- $\alpha$ is considered to have beneficial effects in the treatment of diseases characterized by excess Th2 cells such as UC. In fact, several clinical trials with IFN seem to be successful in chronic active UC $[1,2]$. However, the effect of INF- $\alpha$ on UC remains controversial, because of some reports on the provoking onset and exacerbation of UC by treatment of INF- $\alpha$ [7-9].

In this case, we evaluated the change of Th1/Th2 ratio of CD4 T cells before and after INF- $a$ therapy. The data on intracellular cytokine assay clearly demonstrated that IFN administration increased Th1/Th2 ratio of peripheral CD4 T cells, which was suggestive of shifting cytokine profile toward Th1. In addition, our data showed that administration of PEG-IFN- $\alpha$-2a did not increase IL-10 production from peripheral CD4 T cells. Many mechanisms of effect of IFN therapy on patients with UC have been speculated. Judging from our clinical data, alteration of Th1/Th2 cytokine balance is possible. Thus, alteration 
of Th1/Th2 cytokine balance of peripheral CD4 T cells may be a biomarker for type 1 interferon therapy in patients with UC.

In the future, IFN therapy could be useful for patients with UC refractory to other conventional therapies by practical application of this biomarker.

Fig. 1. a Colonoscopic finding before PEG-IFN- $\alpha$-2a administration showing reddish and edematous mucosa with multiple erosions through the entire colon. $\mathbf{b}$ Colonoscopic finding three months after PEG-IFN- $\alpha-2 a$ administration showing normal colonic appearance.
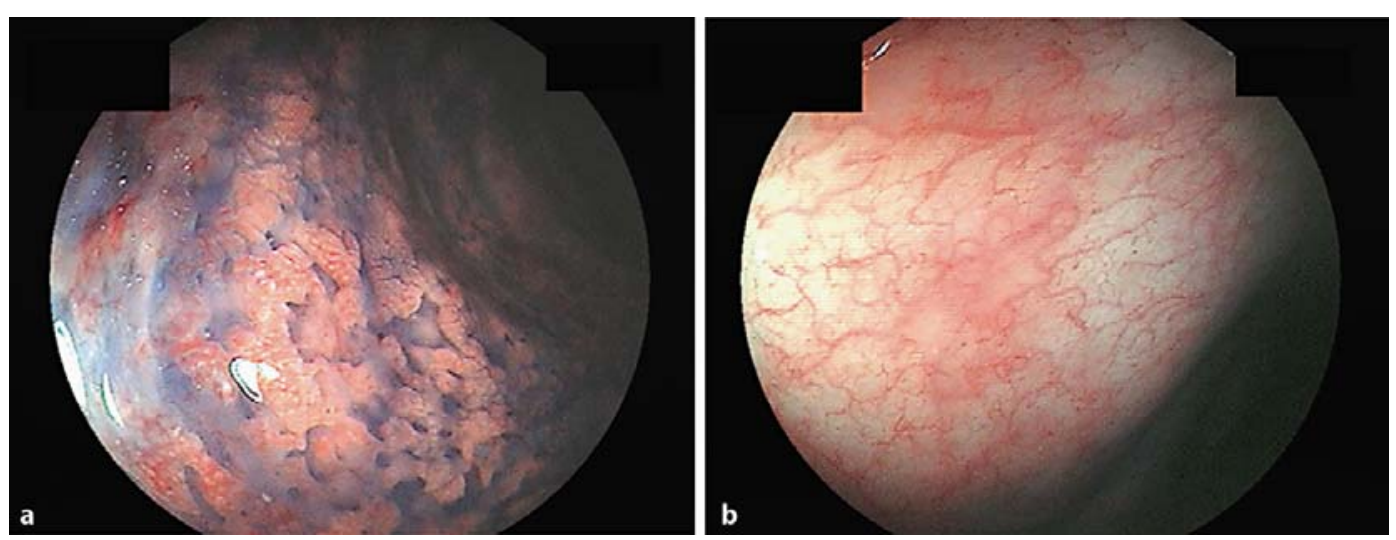
Fig. 2. Comparison of Th1/Th2 ratio of peripheral CD4+ T cells before (a) and after PEG-IFN- $\alpha-2 a$ administration (b). Flow cytometric analysis showing peripheral IFN- $\gamma$ and IL-4 CD4 T cells. Plots showed IL-4-PE on the $y$-axis and IFN- $\gamma$ on the $\mathrm{x}$-axis.
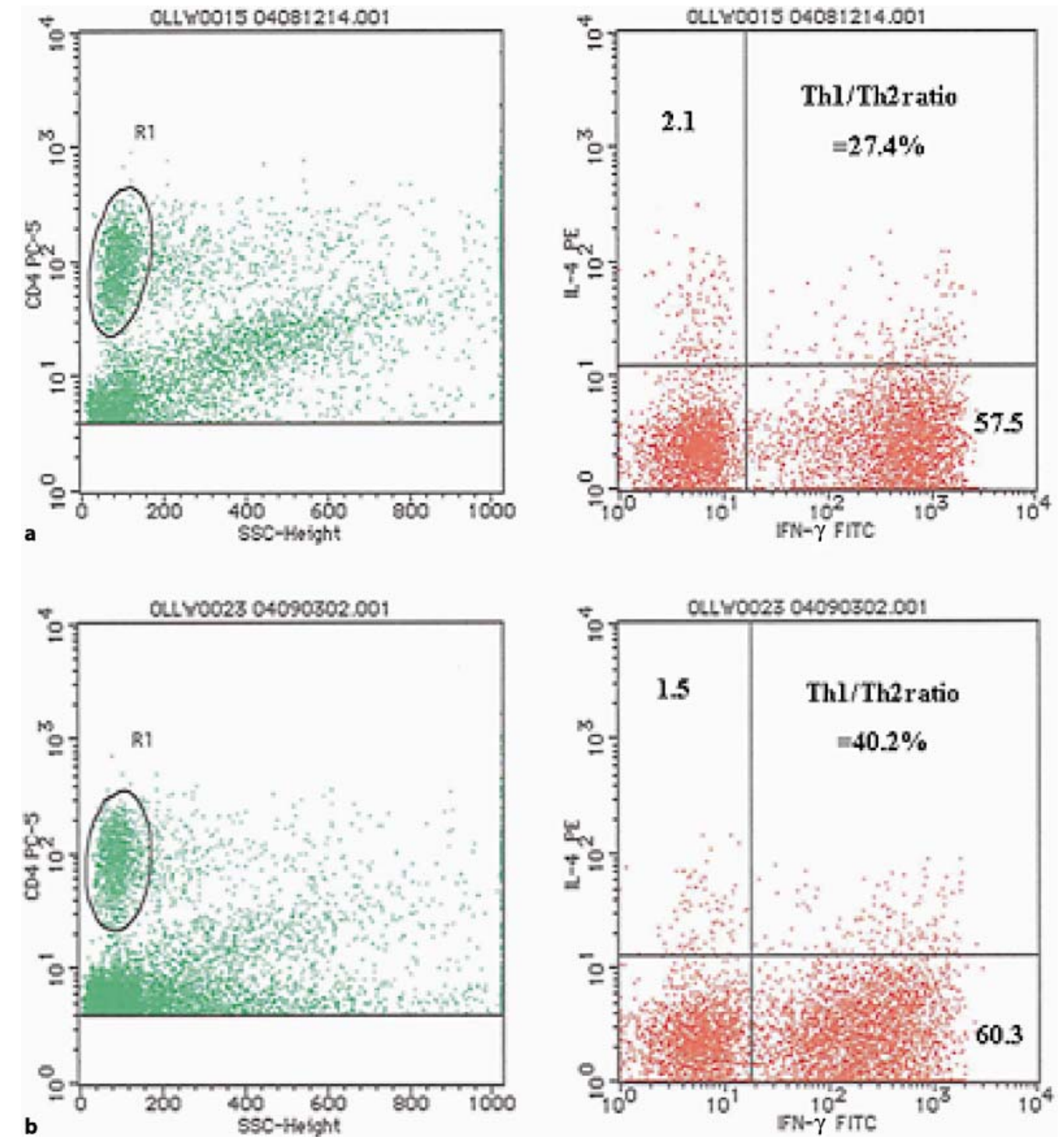


\section{References}

1 Bargiggia S, Thorburn D, Anderloni A, et al: Is interferon-alpha therapy safe and effective for patients with chronic hepatitis $\mathrm{C}$ and inflammatory bowel disease? A case- control study. Aliment Pharmacol Ther 2005;22:209-215.

2 Madsen SM, Schlichting P, Davidsen B, et al: An open-labeled, randomized study comparing systemic interferon-a-2A and prednisolone enemas in the treatment of left-sided ulcerative colitis. Am J Gastroenterol 2001;96:1807-1815.

-3 Musch E, Andus T, Kruis W, et al: Interferon- $\beta$ - 1 a for the treatment of steroidrefractory ulcerative colitis: A randomized, double-blind, placebo controlled trial. Clin Gastroenterol Hepatol 2005;3:581-586.

-4 Tilg H: New insights into the mechanisms of interferon alfa: An immunoregulatory and anti-inflammatory cytokine. Gastroenterology 1997;112:1017-1021.

5 Cammarota G, Cuoco L, Cianci R, et al: Onset of celiac disease during treatment with interferon for chronic hepatitis C. Lancet 2000;356:1494-1495.

6 Khalil A, Lucidarme D, Desurmont P, et al : Crohn's disease associated with interferon and ribavirin treatment for chronic hepatitis C. Gastroenterol Clin Biol 2005;29:193-196.

7 Watanabe T, Inoue M, Harada K, et al : A case of exacerbation of ulcerative colitis induced by combination therapy with PEG-interferon alpha- $2 \mathrm{~b}$ and ribavirin. Gut 2006;55:1682-1683.

8 Tursi A: Rapid onset of ulcerative colitis after treatment with PEG-interferon plus ribavirion for chronic hepatitis C. Inflamm Bowel Dis 2007;13:1189-1190.

-9 Mavrogiannis C, Papanikolaou IS, Elefsiniotis IS, et al: Ulcerative colitis associated with interferon treatment for chronic hepatitis C. J Hepatol 2001;34:964-965. 